\title{
Probing the Variation of the Intervalley Tunnel Coupling in a Silicon Triple Quantum Dot
}

\author{
F. Borjans $\odot,{ }^{1}$ X. Zhang, ${ }^{1}$ X. Mi,,${ }^{1, \dagger}$ G. Cheng $\odot,{ }^{2}$ N. Yao $\odot,{ }^{2}$ C.A.C. Jackson, ${ }^{3}$ L.F. Edge, ${ }^{3}$ and \\ J.R. Petta ${ }^{1}{ }^{1 *}$ \\ ${ }^{1}$ Department of Physics, Princeton University, Princeton, New Jersey 08544, USA \\ ${ }^{2}$ Princeton Institute for Science and Technology of Materials, Princeton University, Princeton, \\ New Jersey 08544, USA \\ ${ }^{3}$ HRL Laboratories LLC, 3011 Malibu Canyon Road, Malibu, California 90265, USA
}

(Received 7 December 2020; accepted 30 March 2021; published 27 April 2021)

\begin{abstract}
Electrons confined in silicon quantum dots exhibit orbital, spin, and valley degrees of freedom. The valley degree of freedom originates from the bulk band structure of silicon, which has six degenerate electronic minima. The degeneracy can be lifted in silicon quantum wells due to strain and electronic confinement, but the "valley splitting" of the two lowest-lying valleys is known to be sensitive to atomic scale disorder. Large valley splittings are desirable to have a well-defined spin qubit. In addition, an understanding of the intervalley tunnel coupling that couples different valleys in adjacent quantum dots is extremely important, as the resulting gaps in the energy-level diagram may affect the fidelity of chargeand spin-transfer protocols in silicon quantum-dot arrays. Here we use microwave spectroscopy to probe variations in the valley splitting, and the intra- and intervalley tunnel couplings $\left(t_{i j}\right.$ and $\left.t_{i j}^{\prime}\right)$ that couple dots $i$ and $j$ in a triple quantum dot. We uncover large variations in the ratio of intervalley to intravalley tunnel couplings $t_{12}^{\prime} / t_{12}=0.90$ and $t_{23}^{\prime} / t_{23}=0.56$. By tuning the interdot tunnel barrier we also show that $t_{i j}^{\prime}$ scales linearly with $t_{i j}$, as expected from theory. The results indicate strong interactions between different valley states on neighboring dots, which we attribute to local inhomogeneities in the silicon quantum well.
\end{abstract}

DOI: 10.1103/PRXQuantum.2.020309

\section{INTRODUCTION}

Continuous research on electron spin qubits defined in silicon quantum dots has led to increasingly impressive levels of quantum control, with recent demonstrations of high single-qubit fidelities [1-3] and $>90 \%$ two-qubit gate fidelities [4,5]. Progress has been fueled by an investment in high-quality $\mathrm{Si} / \mathrm{Si}_{0.7} \mathrm{Ge}_{0.3}$ heterostructures [6,7], coupled with the advent of accumulation-mode device designs that are less sensitive to disorder and enable fine control over quantum-dot electrons [8-11]. For example, through timedomain control of the quantum-dot confinement potential, it is now feasible to shuttle a single charge down an array of nine silicon quantum dots in approximately $50 \mathrm{~ns}$ [12].

\footnotetext{
*petta@princeton.edu

†Present address: Google Inc., Santa Barbara, California 93117, USA.

Published by the American Physical Society under the terms of the Creative Commons Attribution 4.0 International license. Further distribution of this work must maintain attribution to the author(s) and the published article's title, journal citation, and DOI.
}

Control of valley states in Si quantum devices, especially those based on $\mathrm{Si} / \mathrm{Si}_{0.7} \mathrm{Ge}_{0.3}$ heterostructures, is an outstanding challenge [13-15]. While spin-1/2 electrons are often viewed as a canonical two-level system, the valley degree of freedom in the electronic band structure of silicon can give rise to low-lying valley-orbit states [16-18]. For single quantum dots, low-lying valley states inhibit spin initialization and readout routines that are based on energy-dependent tunneling [19]. Moreover, spin-valley mixing leads to spin-relaxation hotspots when the Zeeman splitting of the single-electron spin state is comparable to the valley splitting [20-23].

Complications associated with valley splitting are exacerbated in silicon quantum-dot arrays. The lifting of the $\pm z$-valley degeneracy that gives rise to valley splitting is set by the abruptness of the electronic interfaces that break inversion symmetry [24]. Spatial inhomogeneities in the structure of the quantum well therefore lead to dot-to-dot variations in the valley splitting [25-27]. In $\mathrm{Si} / \mathrm{Si}_{0.7} \mathrm{Ge}_{0.3}$ systems the valley splitting often lies between $25-300 \mu \mathrm{eV}$ [22,28-33]. Due to tighter confinement in the $z$ direction, valley splittings $>200 \mu \mathrm{eV}$ have been observed in Si-MOS systems [34-36]. 
The magnitude of the valley splitting is generally sufficient to understand the consequences on individual spins in isolated quantum dots. However, the intravalley $t_{i j}$ and intervalley $t_{i j}^{\prime}$ tunnel couplings, which depend on the socalled valley-orbit phase, will influence the energy-level structure of a linear array of tunnel-coupled quantum dots. In particular, the location of anticrossings in the energylevel diagram will be set by the valley splitting of each dot $E_{\mathrm{VS}, i}$, and the magnitude of the avoided crossings in the energy-level diagram will be set by $t_{i j}$ and $t_{i j}^{\prime}$ [37-40]. Nonadiabatic transitions, Landau-ZenerStuckelberg-Majorana interference, and leakage into higher-lying energy levels may all influence the fidelity of spin-transfer protocols [41-43]. Moreover, the specific configuration of valley states can strongly affect multielectron exchange coupling in the quantum-dot device [44].

Here we investigate valley splitting and intervalley tunnel coupling using microwave spectroscopy. A triple quantum dot (TQD) is embedded in a superconducting cavity in the circuit quantum electrodynamics device architecture (cQED) [45]. The dipole moment of an electron confined in a quantum dot couples to the electromagnetic field of the superconducting cavity [46-50]. By probing the microwave transmission through the cavity we sensitively map out the energy-level structure of the TQD. To take a first step towards measuring the spatial dependence of valley parameters, we extract the valley splitting of each dot and the intra- and intervalley tunnel couplings in the TQD. We find significant variations in the quantumdot valley parameters across the approximately $200-\mathrm{nm}$ length scale of the device. Valley splittings range from 38-63 $\mu \mathrm{eV}$ and the intervalley tunnel coupling $t_{12}^{\prime}$ between dots 1 and 2 is nearly 2 times larger than $t_{23}^{\prime}$. These results have important implications on future experiments aimed at demonstrating coherent spin shuttling in quantum-dot arrays and reinforce the need for additional improvements in the growth of $\mathrm{Si} / \mathrm{Si}_{0.7} \mathrm{Ge}_{0.3}$ heterostructures, especially interface abruptness.

Beyond providing fundamental insight into the nature of the valley degree of freedom in $\mathrm{Si} / \mathrm{Si}_{0.7} \mathrm{Ge}_{0.3}$ quantum devices, our results pave the way for coherent coupling of resonant-exchange (RX) spin qubits to microwavefrequency photons. Coherent coupling of a single spin to a single photon has been achieved in silicon double quantum dots (DQDs) using a combination of electric dipole coupling and spin-orbit coupling generated by the fringing field of a micromagnet [51-53]. With RX qubits, the electric field of a cavity photon couples to spin through the exchange interaction. High-fidelity gate operations have been demonstrated with electrically tunable RX spin qubits, which can operate at zero magnetic field. This increases compatibility with superconducting cavities [3] and allows for scalable control of the qubit frequency. Strong coupling of a RX spin qubit to a photon has recently been demonstrated in a GaAs TQD [54]. However, due to isotopic enrichment, silicon spin qubits offer much longer spin coherence times and may enable operation deep in the strong coupling regime, once the complications stemming from the presence of valley states are overcome.

\section{VALLEY STATES IN SILICON}

The band structure of bulk silicon has six degenerate conduction-band minima that are located close to the boundary of the first Brillouin zone, as depicted in Fig. 1(a) $[13,55]$. Due to the slight difference in lattice constant of the Si quantum well and the surrounding $\mathrm{Si}_{0.7} \mathrm{Ge}_{0.3}$ buffer layers, the Si quantum well is under tensile strain, which increases the energy of the $\pm x, y$ valleys relative to the $\pm z$ valleys [6]. Sharp quantum-well interfaces break inversion symmetry and couple the two $\pm z$ valleys, resulting in valley splitting [13].

Specifically, the Hamiltonian of the subsystem consisting of the $\pm z$ valleys on dot $i$ can be written in the $\{|i,+z\rangle,|i,-z\rangle\}$ basis as

$$
H_{V, i}=\left(\begin{array}{cc}
0 & \Delta_{i} \\
\Delta_{i}^{*} & 0
\end{array}\right)
$$

with complex valley coupling matrix element $\Delta_{i}=$ $\left|\Delta_{i}\right| e^{-i \phi_{i}}[25,42]$. The eigenstates of this system are
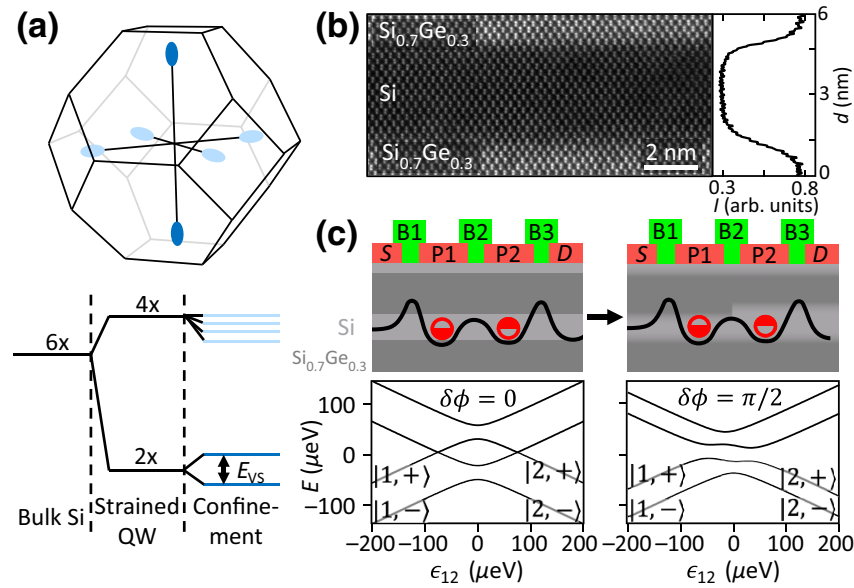

FIG. 1. Valley physics in silicon. (a) First Brillouin zone of silicon, with six degenerate conduction-band minima. Tensile strain in the Si quantum well separates the $\pm z$ valleys from the $\pm x, y$ valleys, while vertical confinement lifts the degeneracy of the $\pm z$ valleys. (b) TEM image of the $\mathrm{Si} / \mathrm{Si}_{0.7} \mathrm{Ge}_{0.3}$ quantum well. (c) Schematic cross section of a DQD with corresponding energy levels. An ideal $\mathrm{Si} / \mathrm{Si}_{0.7} \mathrm{Ge}_{0.3}$ quantum well with abrupt interfaces (left) leads to large and uniform valley splittings, and no intervalley tunnel coupling, while a realistic quantum well with soft interfaces and step edges (right) will have small, nonuniform valley splittings, and strong intervalley tunnel coupling between the quantum dots. Corresponding energy-level diagrams are shown in the bottom graphs. 
$|i, \pm\rangle=(1 / \sqrt{2})\left(|i,+z\rangle \pm e^{i \phi_{i}}|i,-z\rangle\right)$, with eigenenergies $E_{ \pm}= \pm\left|\Delta_{i}\right|$. Consequently, the valley splitting of $\operatorname{dot} i$ is $E_{\mathrm{VS}, i}=E_{+}-E_{-}=2\left|\Delta_{i}\right|$ and $\phi_{i}$ is the valley-orbit phase. The magnitude and phase of $\Delta_{i}$ are physically rooted in the local properties of the quantum well $[25,42]$. For example, spatial variations in interface abruptness and disorder will cause $\Delta_{i}$ to be a function of position in the plane of the quantum well, $\Delta_{i}=\Delta_{i}(x, y)$, hence the term "valley-orbit coupling."

While the valley-orbit phase does not immediately affect the energy levels of a single quantum dot, its consequences are evident when considering a system of two tunnel coupled quantum dots (dots $i$ and $j$ ). In the basis $\{|i,+z\rangle,|i,-z\rangle,|j,+z\rangle,|j,-z\rangle\}$, the Hamiltonian can be expressed as

$$
H_{i j}\left(\epsilon_{i j}\right)=\left(\begin{array}{cccc}
\frac{\epsilon_{i j}}{2} & \Delta_{i} & t_{c} & 0 \\
\Delta_{i}^{*} & \frac{\epsilon_{i j}}{2} & 0 & t_{c} \\
t_{c} & 0 & -\frac{\epsilon_{i j}}{2} & \Delta_{j} \\
0 & t_{c} & \Delta_{j}^{*} & -\frac{\epsilon_{i j}}{2}
\end{array}\right),
$$

with tunnel coupling $t_{c}$ between identical $z$-valleys of dots $i$ and $j$. We neglect the coupling of opposing $z$ valleys between the quantum dots, as it is a higher-order coupling contribution [25]. The energy-level detuning between dots $i$ and $j$ is defined by $\epsilon_{i j}=\mu_{i}-\mu_{j}$ with corresponding decoupled chemical potentials $\mu_{i(j)}$.

We diagonalize the local single-dot valley dynamics of dots $i$ and $j$ by transforming into the basis $\{|i,+\rangle,|i,-\rangle,|j,+\rangle,|j,-\rangle\}$, where \pm refers to the bonding and antibonding valley state combinations. We obtain

$$
H_{i j}^{\prime}\left(\epsilon_{i j}\right)=\left(\begin{array}{cccc}
\frac{\epsilon_{i j}}{2}+E_{\mathrm{VS}, i} & 0 & t_{i j} & t_{i j}^{\prime} \\
0 & \frac{\epsilon_{i j}}{2} & t_{i j}^{\prime} & t_{i j} \\
t_{i j}^{*} & t_{i j}^{* *} & -\frac{\epsilon_{i j}}{2}+E_{\mathrm{VS}, j} & 0 \\
t_{i j}^{\prime *} & t_{i j}^{*} & 0 & -\frac{\epsilon_{i j}}{2}
\end{array}\right),
$$

with intra- and intervalley tunnel coupling $t_{i j}=(1 / 2) t_{c}(1+$ $\left.e^{-i \delta \phi_{i j}}\right)$ and $t_{i j}^{\prime}=(1 / 2) t_{c}\left(1-e^{-i \delta \phi_{i j}}\right)$, and valley phase difference $\delta \phi_{i j}=\phi_{i}-\phi_{j}[42,56]$. This Hamiltonian results in four energy bands with two intravalley and two intervalley anticrossings determined by $t_{i j}$ and $t_{i j}^{\prime}$, respectively.

In practice, the valley splitting and phase are strongly dependent on the valley coupling dynamics at the $\mathrm{Si} / \mathrm{Si}_{0.7} \mathrm{Ge}_{0.3}$ quantum-well interfaces. Figure 1(b) shows a high-resolution TEM image of the heterostructure. The varying Ge content causes an intensity contrast between the different layers of the heterostructure. To the right, we plot the average of the grayscale pixel intensity for each row of the image. The interface is of fairly high quality. This is expressed by the smooth transition between the average intensities for $\mathrm{Si}$ and $\mathrm{Si}_{0.7} \mathrm{Ge}_{0.3}$ only spanning a few $(\leq 5)$ lattice sites over the displayed range. However, even a small number of atomic steps in the interface can have a large impact on the valley phase difference and overall structure of the DQD energy-level diagram $[16,24,37,39]$. A uniform (step-edge-free) interface in the region of the DQD corresponds to the limit $\delta \phi_{i j}$ $=0$, which results in a set of bonding-antibonding charge states for each of the valleys [see Fig. 1(c), left panel]. Realistic quantum wells will have less abrupt interfaces and step edges that result in intervalley tunnel coupling [see Fig. 1(c), right panel]. For example, with $\delta \phi_{i j}=\pi / 2$, there are four avoided crossings in the energy-level diagram, indicating the presence of both valley-conserving and nonconserving charge transitions between dots $i$ and $j$. Moreover, smooth, disordered interfaces will generally yield smaller $E_{\mathrm{VS}}$ that exhibit dot-to-dot variations [25]. Comprehensive studies of the full set of valley parameters have so far not been conducted. Microwave spectroscopy of a TQD in the CQED architecture provides an opportunity to sensitively probe the spatial variation of valley states in a silicon quantum device.

\section{EXPERIMENTAL SETUP}

In this experiment a half-wavelength $\lambda / 2$ superconducting cavity with resonance frequency $f_{c}=6.76 \mathrm{GHz}$ and photon loss rate $\kappa / 2 \pi=1.4 \mathrm{MHz}$ is coupled to two $\mathrm{Si} / \mathrm{Si}_{0.7} \mathrm{Ge}_{0.3}$ TQDs [Fig. 2(a)]. The TQDs are fabricated using an overlapping gate architecture [29]. The center pin of the cavity is galvanically connected to the cavitycoupler gate "CP," as shown schematically in Fig. 2(b). Along with screening gates $\mathrm{S} 1$ and $\mathrm{S} 2$, the $\mathrm{CP}$ gate is part of the first of three overlapping Al gate layers [57]. Plunger (barrier) gates are defined in the second (third) aluminum layers and the layers are electrically isolated from one another by a native $\mathrm{Al}_{2} \mathrm{O}_{3}$ oxide barrier.

The TQD chemical potentials are controlled with plunger gates $\mathrm{P} 1-\mathrm{P} 3$, while the interdot barriers and barriers to the source $(S)$ and drain $(D)$ reservoirs are controlled by gates B1-B4. To enhance the dot-cavity coupling, the $\mathrm{CP}$ gate is designed to wrap around dot 3 , which effectively adds a potential barrier between dot 3 and the $D$ reservoir, in addition to the barrier defined by gate B4. A SEM image of the TQD is shown in Fig. 2(c), with a white dashed line indicating the long axis of the TQD. Details related to the fabrication of similar devices have been presented elsewhere [58]. The data sets in this paper are acquired from the TQD located at the right antinode of the cavity (denoted RTQD).

We map out the TQD charge stability diagram [Fig. 2(d)] by measuring the normalized cavity transmission $A / A_{0}$ as a function of the gate voltages $V_{\mathrm{P} 1}$ and $V_{\mathrm{P} 3}$ [46]. When sweeping $V_{\mathrm{P} 1}$ and $V_{\mathrm{P} 3}$ we observe nearly horizontal charge transitions in the cavity response that 


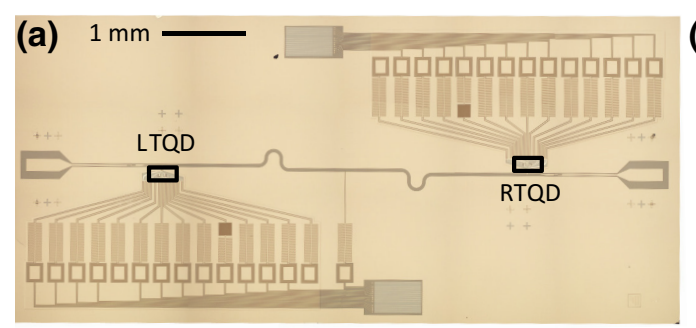

(b)

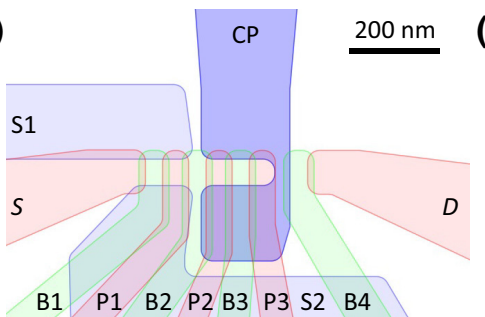

(e)

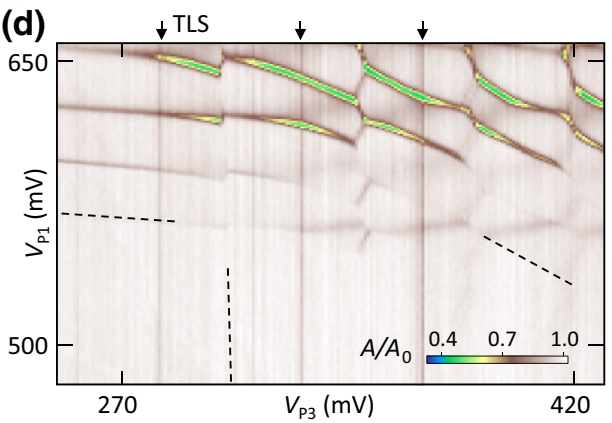

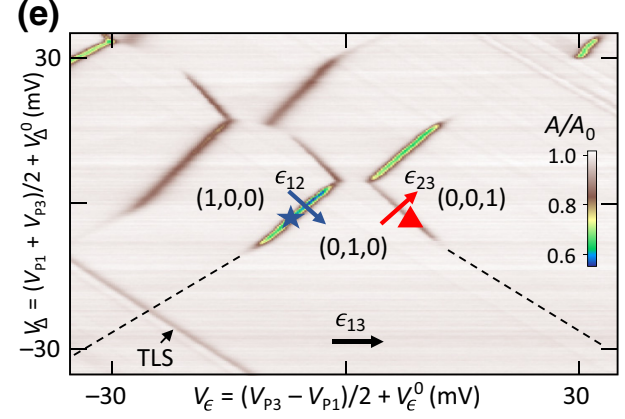

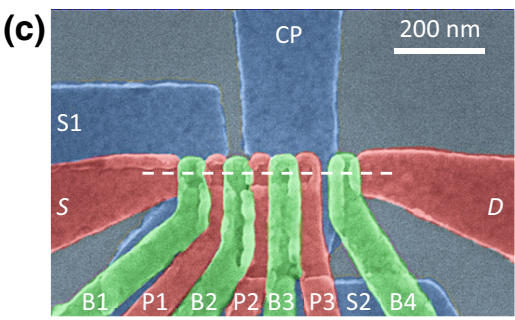

(f)

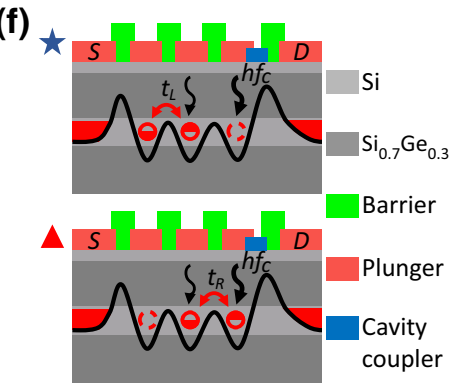

FIG. 2. Cavity-coupled TQD. (a) Optical image of a superconducting cavity coupled to two TQDs. (b) Schematic of the TQD device with cavity coupler gate (CP). (c) False-color SEM image of the TQD. (d) Large-scale charge stability diagram of the TQD. The dashed black lines indicate the three quantum-dot charge transitions. Features corresponding to two-level systems (TLSs) are indicated by black arrows. (e) Charge stability diagram in the single-electron regime. The blue star indicates the $(1,0,0)-(0,1,0)$ interdot transition and the red triangle indicates the $(0,1,0)-(0,0,1)$ interdot transition. (f) Schematic of the operating points indicated by the symbols in (e). Due to the geometry of CP, the microwave coupling to dot 3 is strongest, as indicated by the thickness of the black arrows.

correspond to charge transfer between dot 1 and the $S$ reservoir. As a result of the mutual charging energy, the dot- 1 charge transitions shift abruptly when an electron is added or removed from dot 3 (see vertical dashed line). As the $\mathrm{CP}$ gate limits the tunneling rate to the $D$ reservoir in the few-electron regime, no direct cavity response is observed for dot-3 charge transitions. The absence of dot-1 transition shifts for $V_{\mathrm{P} 3}$ below the indicated vertical dashed line however allows us to verify the single-electron occupation of dot 3. Due to cross-coupling of the gate voltages $V_{\mathrm{P} 1}$ and $V_{\mathrm{P} 3}$ to dot 2, we observe angled dot-2 charge transitions in the data, which interact with the dot-1 and dot-3 charging transitions.

For the remainder of the paper, we operate in the single-electron regime. By performing pairwise sweeps of $V_{\mathrm{P} 1} / V_{\mathrm{P} 2}$ and $V_{\mathrm{P} 2} / V_{\mathrm{P} 3}$, we tune the device towards the $(1,0,0)-(0,1,0)-(0,0,1)$ transition shown in Fig. 2(e) (see Appendix A). Here we use the virtual gates $V_{\epsilon}=\left(V_{\mathrm{P} 3}-\right.$ $\left.V_{\mathrm{P} 1}\right) / 2+V_{\epsilon}^{0}$ and $V_{\Delta}=\left(V_{\mathrm{P} 1}+V_{\mathrm{P} 3}\right) / 2+V_{\Delta}^{0}$ to access the different charge states of the TQD. Voltage offsets $V_{\epsilon}^{0}$ and $V_{\Delta}^{0}$ are used to move the $(0,1,0)$ charge state to the origin. In this tuning configuration, the tunneling rates to the $S$ and $D$ reservoirs are suppressed relative to $f_{c}$, and the primary cavity response stems from the $(1,0,0)-(0,1,0)$ and $(0,1,0)-(0,0,1)$ interdot charge transitions. We denote these transitions with a blue star and a red triangle, respectively, and show a schematic of the device cross section in Fig. 2(f). The cross section corresponds to the device area indicated by the white dashed line in Fig. 2(c). The asymmetric geometry of the $\mathrm{CP}$ gate creates an electric field gradient across all three dots, allowing us to observe a signal at both interdot charge transitions.

\section{CAVITY RESPONSE TO VALLEY STATES}

To gather insight into the TQD valley physics we sketch the low-lying TQD energy levels as a function of the detuning $\epsilon_{13}$ between dots 1 and 3 in Fig. 3(a). In this regime the $(1,0,0)-(0,1,0)$ and $(0,1,0)-(0,0,1)$ interdot charge transitions can be analyzed independently. Each interdot transition involves four energy levels. Focusing on the $(1,0,0)-(0,1,0)$ transition the four relevant levels are $|1, \pm\rangle$ and $|2, \pm\rangle$.

The microwave cavity is sensitive to charge dynamics within the TQD due to dipole coupling between the electron trapped in the device and the cavity electric field [46,47]. In general, the cavity response $A / A_{0}$ is strongest near charge avoided crossings in the energy-level diagram [see blue, red, and green arrows in Fig. 3(a)]. At low temperatures where $k_{B} T_{e} \ll E_{\mathrm{VS}, i}$, with Boltzmann constant $k_{B}$ and electron temperature $T_{e}$, the electron primarily resides in the ground state and the excited valley states are not prominent in the cavity response. Participation of the excited valley states $|1,+\rangle,|2,+\rangle,|3,+\rangle$ in the cavity response can be increased by raising the temperature and thermally exciting the charge according to the Boltzmann distribution with occupation probability $p_{i}=e^{-E_{i} / k_{B} T_{e}} / \sum_{i} e^{-E_{i} / k_{B} T_{e}}$, with state energy $E_{i}$ $[30,56]$. Increasing the electron temperature, such that 


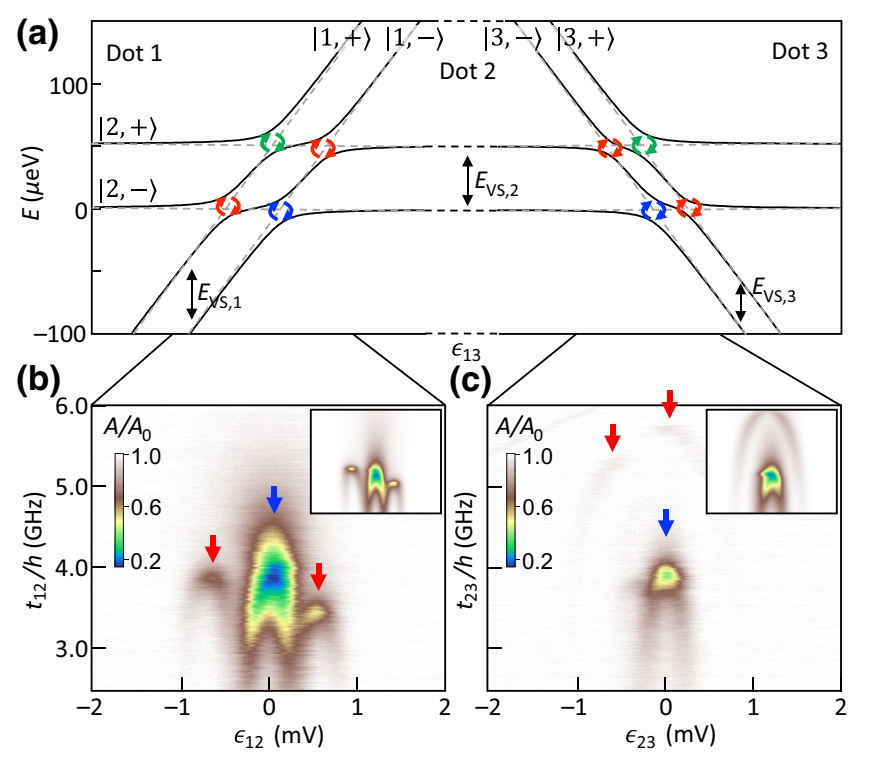

FIG. 3. Cavity response in the single-electron regime at $T_{e}=$ $350 \mathrm{mK}$. (a) Energy bands of the TQD, with valley splittings denoted by $E_{\mathrm{VS}, i}$. At low temperatures, only the transition between the lowest energy states (blue) is visible in the cavity response. By increasing temperature, intervalley transitions (red) can be accessed. (b) Cavity response $A / A_{0}$ near the $(1,0,0)$ $(0,1,0)$ transition as a function of $t_{12}$ and the detuning $\epsilon_{12}$ between dots 1 and 2. The colored arrows correspond to the transitions highlighted in (a). (c) $A / A_{0}$ in the vicinity of the $(0,1,0)-(0,0,1)$ transition as a function of $t_{23}$ and $\epsilon_{23}$. Insets in (b),(c) show the simulated cavity response.

$k_{B} T_{e} \approx E_{\mathrm{VS}, i}$, is effective at increasing the visibility of the higher-lying intervalley transitions in the cavity response (red transitions). In practice, the excited intravalley transitions (green transitions) are still masked by the larger participation of the ground-state intravalley transitions at nearly the same voltage and are therefore not visible here. Nevertheless, the cavity-response data are sufficient to extract the valley splittings, and intra- and intervalley tunnel couplings.

Figure 3(b) displays the cavity transmission $A / A_{0}$ as a function of the interdot detuning $\epsilon_{12}$ and tunnel coupling $\left|t_{12}\right|$ with $T_{e}=350 \mathrm{mK}$, which is achieved by actively heating the mixing chamber plate of the dilution refrigerator (see Appendix B). Here the detuning is plotted in units of $\mathrm{mV}$, as the lever arm varies slightly as a function of $t_{12}$. The ground-state transition $|1,-\rangle \leftrightarrow|2,-\rangle$ corresponds to the center arch that is most pronounced in the data. With $\epsilon_{12} \approx 0$, the cavity response is the strongest around $t_{12} / h=4 \mathrm{GHz}$ ( $h$ is Planck's constant), where the energy difference of the coupled states is most insensitive to noise on the detuning axis $\epsilon_{12}$ and the transition energy is resonant with the cavity $E_{2}-E_{1}=h f_{c}$. In contrast to the valley-free case [50,57], here $E_{2}-E_{1}<2 t_{12}$, as level repulsion from the excited valley states $|1,+\rangle$ and $|2,+\rangle$ reduce its effective energy splitting.

Next to the strong central feature we observe two similar, but fainter, arches. These arches correspond to the thermally occupied intervalley transitions $|1, \pm\rangle \leftrightarrow|2, \mp\rangle$ (red arrows). We note that tuning $t_{12}$ by adjusting $V_{\mathrm{B} 2}$ affects both the inter- and intravalley signatures in the data. Moreover, the position of the side arches can be qualitatively understood by looking at the energy diagram in Fig. 3(a). Raising $E_{\mathrm{VS}, 1}$ will move the $|1,+\rangle \leftrightarrow|2,-\rangle$ transition towards more negative detuning. Similarly, $E_{\mathrm{VS}, 2}$ affects the horizontal positioning of the right-side arch via a shift of the $|1,-\rangle \leftrightarrow|2,+\rangle$ transition. From this qualitative analysis and the larger spacing between the left and center feature, we deduce $E_{\mathrm{VS}, 1}>E_{\mathrm{VS}, 2}$. To understand the vertical positioning of the arches, we focus on the strongest cavity signal at the maxima of the arches indicated by the blue (red) arrows. At these points the intra(inter)valley transition energies are dominated by the contribution of the corresponding couplings $t_{12}\left(t_{12}^{\prime}\right)$ and coincide with $h f_{c}$. The observation that these points are reached at different values of $t_{12}$ for the two side arches indicates that in fact $t_{12}^{\prime}$ changes with detuning in our system, i.e., $t_{12}^{\prime}=t_{12}^{\prime}(\epsilon)$. This suggests that also $t_{12}=t_{12}(\epsilon)$ due to their functional interdependence. Specifically, with $t_{12}(\epsilon=0) / h=3.5 \mathrm{GHz}, t^{\prime}$ is large enough for the $|1,-\rangle \leftrightarrow|2,+\rangle$ transition to be resonant at positive detuning, while it is too small at negative detuning leaving the $|1,+\rangle \leftrightarrow|2,-\rangle$ transition below resonance. Unless the detuning dependence is explicitly specified, we identify $t_{i j}=t_{i j}(\epsilon=0)$ and $t_{i j}^{\prime}=t_{i j}^{\prime}(\epsilon=0)$. We assume that this detuning dependence is caused by cross capacitance between the plunger gates and the tunnel barrier potential.

For the $(0,1,0)-(0,0,1)$ transition [Fig. 3(c)], we observe similar behavior for the $|2,-\rangle \leftrightarrow|3,-\rangle$ intravalley transition. However, the $|2, \pm\rangle \leftrightarrow|3, \mp\rangle$ intervalley features do not appear in the shape of two arches next to the main feature, but they merge into one bigger arch at much higher $t_{23}$. The qualitative differences between the datasets indicates a variation of the valley parameters across the TQD. The asymmetries caused by the variation of $t$ and $t^{\prime}$ can be reproduced by input-output simulations shown in the insets of Figs. 3(b) and 3(c) [59].

\section{QUANTITATIVE EXTRACTION OF THE VALLEY PARAMETERS}

We now quantitatively analyze the cavity response data in light of the theoretical background given in Sec. II. The energy levels in the vicinity of the $(1,0,0)-(0,1,0)$ transition are plotted in Fig. 4(a). The dashed lines indicate the expected energy levels with no tunnel coupling, while the solid lines show the energy levels with $\left|t_{12}\right| / h=2.9 \mathrm{GHz}$ and $\left|t_{12}^{\prime}\right| / h=2.6 \mathrm{GHz}$. We denote the intravalley transition $|1,-\rangle \leftrightarrow|2,-\rangle$ as (i), and the intervalley transitions 
(a)

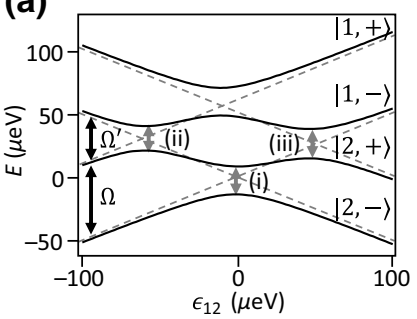

(c)

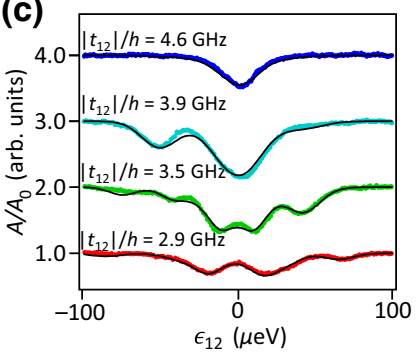

(b)

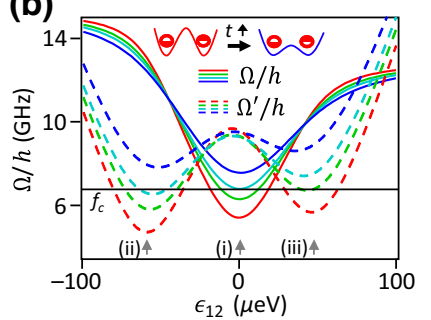

(d)

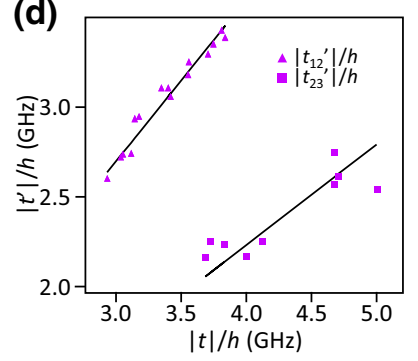

FIG. 4. Quantitative analysis of the cavity response. (a) Energy-level diagram for $\left|t_{12}\right| / h=2.9 \mathrm{GHz}$. Transitions $\Omega$ and $\Omega^{\prime}$ are energetically accessible at $T_{e}=350 \mathrm{mK}$. (b) Transition frequencies $\Omega / h$ and $\Omega^{\prime} / h$ as a function of $\epsilon_{12}$ for the four values of $t_{12}$ indicated in (c). The minima in the transition frequencies are labeled (i)-(iii). (c) Line cuts extracted from Fig. 3(b) for different $|t|$ (fits are shown in black). The line cuts correspond to the low $|t|$ regime, resonant regimes for both intra- and intervalley transitions, and the high $|t|$ regime. (d) Extracted intervalley tunnel coupling rates $\left|t_{i j}\right| / h$ as a function of $\left|t_{i j}\right| / h$ for both interdot transitions.

$|1, \pm\rangle \leftrightarrow|2, \mp\rangle$ as (ii) and (iii), respectively. We plot the intra- and intervalley transition frequencies $\Omega / h$ and $\Omega^{\prime} / h$ as a function of $\epsilon_{12}$ in Fig. 4(b), and the cavity frequency $f_{c}$ is shown for comparison. An appreciable cavity response is observed near detunings $\epsilon_{12}$ where $\Omega / h$ or $\Omega^{\prime} / h \approx f_{c}$. $\Omega / h$ exhibit a single minimum corresponding to the groundstate intravalley transition (i) close to $\epsilon=0$. Similarly, the two intervalley transitions (ii) and (iii) lead to two minima in $\Omega^{\prime} / h$ at finite detuning.

We next utilize Figs. 4(a) and 4(b) to identify the features in the cavity-response data. Four linecuts extracted from Fig. 3(b) data set are plotted in Fig. 4(c), and show the evolution of $A / A_{0}$ as a function of $\epsilon_{12}$ as $t_{12}$ is reduced. Qualitatively, the complexity of the cavity response increases as $\left|t_{12}\right| / h$ is reduced from 4.6 to $2.9 \mathrm{GHz}$. At $\left|t_{12}\right| / h=4.6 \mathrm{GHz}$, both transition energies lie above resonance for the whole range of $\epsilon_{12}$, with $\Omega / h$ only approaching $f_{c}$ at $\epsilon_{12}=0$. At this point, the intervalley features are very weak and only the intravalley transition (i) contributes to an appreciable dispersive signal in the cavity transmission. At $\left|t_{12}\right| / h=3.9 \mathrm{GHz}$, both (i) and (ii) are nearly resonant with the cavity, leading to a strong suppression of $A / A_{0}$ at $\epsilon_{12}=0$, and a local minimum at negative $\epsilon_{12}$. Reducing the tunnel coupling further to $\left|t_{12}\right| / h=3.5 \mathrm{GHz}$, (iii) is nearly resonant with

$f_{c}$, causing a pronounced reduction of the cavity transmission at positive detuning. As (i) is below resonance, we observe two additional minima in $A / A_{0}$, corresponding to the two detuning values where $\Omega / h=f_{c}$. Finally, when $\left|t_{12}\right| / h=2.9 \mathrm{GHz}$, the ground-state intravalley transition (i) and both intervalley transitions (ii) and (iii) lie below resonance, so that there are in theory six detuning values at which either $\Omega / h$ or $\Omega^{\prime} / h=f_{c}$. However, the central four resonance conditions lie pairwise close to each other, such that their corresponding features merge into two central transmission minima [see lower portion of Figs. 3(b), and 4(c)].

To determine the TQD valley parameters we quantitatively analyze linecuts similar to those shown in Fig. 4(c). Superimposed on the data are best fits obtained by numerically diagonalizing the Hamiltonian in Eq. (3) for each value of $\epsilon_{12}$ and feeding the resulting energies into cavity input-output theory, where the cavity signal is calculated based on the coherent coupling of the cavity to the delocalized electron and the decoherence of the coupled systems [60]. To generate the level diagram, we treat $t_{12}=\left|t_{12}\right|$ and $t_{12}^{\prime}=\left|t_{12}^{\prime}\right|$ as real valued parameters for the fits, which leaves the energy levels unaffected $[42,56]$. To account for the observed asymmetry in Fig. 3(b) intervalley tunnel couplings at positive and negative detuning, we parameterize a small linear dependence of the intravalley coupling on detuning $t_{i j}\left(\epsilon_{i j}\right)=t_{i j}\left(\epsilon_{i j}=0\right)+a_{i j} \epsilon_{i j}$. Fit parameters of the Hamiltonian are $E_{\mathrm{VS}, i}, t_{i j}\left(\epsilon_{i j}=0\right), a_{i j}$, leverarm $\alpha_{i j}$ and the valley phase difference $\delta \phi_{i j}$, which is defined by $\left|t_{i j}^{\prime} / t_{i j}\right|=\tan \left(\delta \phi_{i j} / 2\right)$. We take into account the finite population of the excited states at $T_{e}=350 \mathrm{mK}$ via the Boltzmann distribution. In addition, we fit the charge-cavity coupling rate $g_{i j} / 2 \pi$. The inhomogenous broadening of the features caused by charge noise is implemented by a Gaussian convolution of standard deviation $\sigma_{i j}$. Combining the results from the linecut fits we find $g_{12} / 2 \pi=37 \mathrm{MHz}$ and $\sigma_{12}=9 \mu \mathrm{eV}$. The charge decoherence rate $\gamma_{12} / 2 \pi=$ $32 \mathrm{MHz}$ is extracted in a separate measurement by probing the ESR linewidth. Similar data (not shown) are acquired near the $(0,1,0)-(0,0,1)$ transition, with best-fit parameters $g_{23} / 2 \pi=23 \mathrm{MHz}, \gamma_{23} / 2 \pi=39 \mathrm{MHz}$, and $\sigma_{23}=7 \mu \mathrm{eV}$.

We first extract the valley splitting of each quantum dot. Analyzing the linecuts yields $E_{\mathrm{VS}, 1}=63 \mu \mathrm{eV}$ and $E_{\mathrm{VS}, 2}=$ $53 \mu \mathrm{eV}$, whereas the data from the $(0,1,0)-(0,0,1)$ transition yields $E_{\mathrm{VS}, 2}=50 \mu \mathrm{eV}$ and $E_{\mathrm{VS}, 3}=38 \mu \mathrm{eV}$. The magnitude of the valley splitting is consistent with previous measurements on similar devices [30]. Crucially, the values of $E_{\mathrm{VS}, 2}$ obtained from the two independent measurements are self-consistent and imply that the valley splittings are insensitive to small variations in the plunger gate voltages.

Next, we extract values of $\left|t_{12}^{\prime}\right|$ for multiple values of $\left|t_{12}\right|$, as shown in Fig. 4(d). We expect a linear relationship between $\left|t_{i j}^{\prime}\right|$ and $\left|t_{i j}\right|$ described by the formula $\left|t_{i j}^{\prime} / t_{i j}\right|=\tan \left(\delta \phi_{i j} / 2\right)$. We determine the scaling relation 

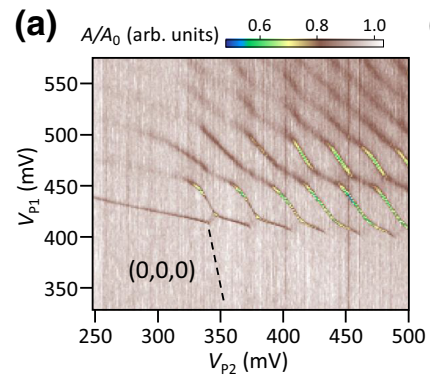

(b) $A / A_{0}$ (arb. units) $\begin{array}{llll}0.4 & 0.6 & 0.8 & 1.0\end{array}$

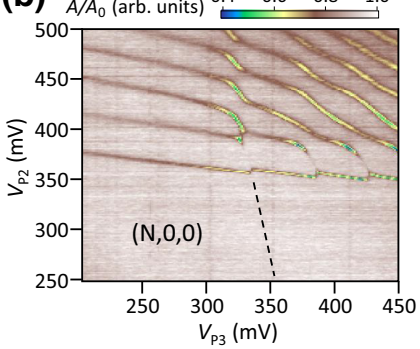

FIG. 5. Single-electron occupation of the TQD. (a) Cavity transmission $A / A_{0}$ as a function of plunger gate voltages $V_{\mathrm{P} 1}$ and $V_{\mathrm{P} 2}$, showing charge stability diagram of dots 1 and 2 with dot 3 empty. (b) $A / A_{0}$ as a function of $V_{\mathrm{P} 2}$ and $V_{\mathrm{P} 3}$, showing charge stability diagram of dots 2 and 3 with dot 1 accumulated to extend the reservoir.

to be $\left|t_{12}^{\prime} / t_{12}\right|=0.90$ for the $(1,0,0)-(0,1,0)$ transition with $a_{12}=0.02$. Similarly, we extract $\left|t_{23}^{\prime} / t_{23}\right|=0.56$ for the $(0,1,0)-(0,0,1)$ transition with $a_{23}=0$. Using these scaling relationships, we find valley phase differences $\delta \phi_{12}=$ $84^{\circ}$ and $\delta \phi_{23}=58^{\circ}$. The substantial valley phase differences point toward a level diagram closely resembling that shown in Fig. 4(a), with the intra- and intervalley tunnel couplings leading to four avoided crossings. In contrast, an abrupt and uniform $\mathrm{Si} / \mathrm{Si}_{0.7} \mathrm{Ge}_{0.3}$ interface would yield $\delta \phi_{12}=0$, with only nonzero intravalley couplings [left panel of Fig. 1(c)].

\section{CONCLUSION AND OUTLOOK}

In conclusion, we use microwave spectroscopy to probe the energy-level structure of a Si TQD containing a single electron. Consistent with previous work, we find significant variations in the valley splitting across the device [22,28-32]. Going beyond previous work, we capitalize on the sensitivity of $\mathrm{cQED}$ microwave spectroscopy to the curvature of the quantum-dot energy levels to probe variations in the intervalley tunnel coupling. Consistent with theory, we find that the intervalley coupling scales linearly with the intravalley coupling. Moreover, examination of both the $(1,0,0)-(0,1,0)$ and $(0,1,0)-(0,0,1)$ interdot charge transitions allows us to probe variations in the valley-orbit phase. Significant variations in the valley phase difference $\delta \phi_{i j}$ over the small approximately $200 \mathrm{~nm}$ length scale of the device highlights the importance of improving the $\mathrm{Si} / \mathrm{Si}_{0.7} \mathrm{Ge}_{0.3}$ interface quality. We expect that experimental characterizations similar to those performed here may be achieved with reflectometry setups utilizing external resonant circuits [60].

Our results indicate a significant variation of the valley splitting and valley-orbit phase across the TQD. Variable valley splittings can impact single-qubit operation and readout, as rapid spin-valley mixing occurs when the
Zeeman energy and valley splitting are comparable, resulting in vastly reduced spin liftetimes [34]. Small valley splittings also limit the use of Pauli spin blockade for singlet-triplet readout in silicon [61]. With larger valley splitting, a device as studied here will also allow the coupling of a resonant exchange-based encoded spin qubit to a superconducting cavity, opening the door to scalable spin-photon links.

Considering efforts to transport spins down large quantum-dot arrays [12], spatial variations in the valley phase difference $\delta \phi_{i j}$ could be especially problematic. From the Landau-Zener model, the nonadiabatic transition probability at an avoided crossing is exponentially dependent on the square of the coupling matrix element. Ideally, the intervalley gaps in the level diagram would be zero or small, limiting mixing into excited valley states during spin shuttling. The nonzero valley phase difference, and its variation across the device, could very well impact the performance of spin-shuttling protocols. Looking forward, the measurements presented here can be used to provide valuable feedback on the quality of interfaces in the $\mathrm{Si} / \mathrm{Si}_{0.7} \mathrm{Ge}_{0.3}$ system.

\section{ACKNOWLEDGMENTS}

We thank Guido Burkard and Mark Friesen for critical comments on the paper, and Michael Gullans and Stefan Putz for technical contributions to the research. Supported by Army Research Office Grant No. W911NF15-1-0149 and the Gordon and Betty Moore Foundation's EPiQS Initiative through Grant No. GBMF4535. Devices are fabricated in the Princeton University Quantum Device Nanofabrication Laboratory, which is managed by the Department of Physics. The authors acknowledge the use of Princeton's Imaging and Analysis Center, which is partially supported by the Princeton Center for Complex Materials, a National Science Foundation MRSEC Program (DMR-2011750).

\section{APPENDIX A: CHARGE STABILITY DIAGRAMS}

In this appendix we show additional data exemplifying the pairwise sweeps used to achieve the single-electron regime in the TQD. As the dot 3 to drain transition is opaque, we operate using the dot 1 to source transition and the capacitive interdot interactions to determine the charge occupation in the array. In Fig. 5(a) we show the charge stability diagram of dots 1 and 2 while keeping dot 3 empty. We increase $V_{\mathrm{B} 1}$ to allow for fast tunneling between the source lead and dot 1 . The first dot- 1 electron transition is clearly visible, with the higher transition becoming smoother as the tunneling rate increases. From the absence of capacitive jumps in the dot- 1 transition, we can infer the last electron transition of dot 2 , indicated with the vertical black dashed line. 
(a)

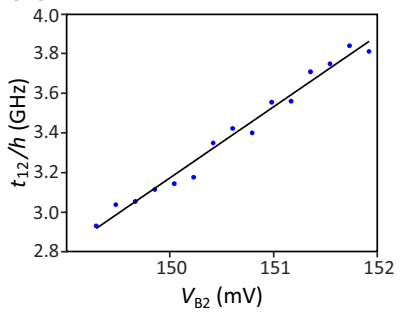

(b)

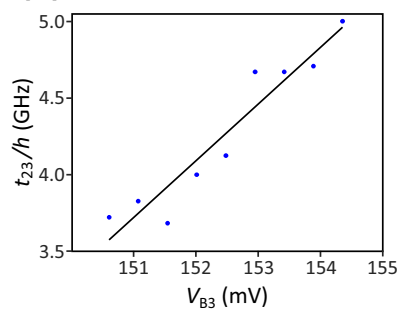

FIG. 6. Tunnel coupling calibration in the TQD. (a) $t_{12}$ is extracted as a function of $V_{\mathrm{B} 2}$ by fitting the input-output model described in the main text to line cuts of Fig. 3(b) data. (b) Similarly, $t_{23}$ is extracted as a function of $V_{\mathrm{B} 3}$ using line cuts of Fig. 3(c) data.

To access the single-electron regime for dots 2 and 3, we accumulate dot 1 such that we can now see the extended source to dot-2 transition in Fig. 5(b). This allows us to directly find the last electron in dot 2 and again infer the last dot 3 transition by the missing capacitive shifts in the dot-2 transition for lower $V_{\mathrm{P} 3}$. Here, the vertical black dashed line indicates the last electron transition for dot 3. Combining the information gathered from these pairwise sweeps, we tune the TQD into the single-electron regime.

\section{APPENDIX B: CALIBRATING THE INTERDOT TUNNEL COUPLING}

We show the calibration of the intravalley tunnel couplings used in Figs. 3(b) and 3(c) in this appendix. The data for these figures are taken by stepping the barrier gate voltages $V_{\mathrm{B} 2}\left(V_{\mathrm{B} 3}\right)$ (vertical axis) and sweeping the detuning (horizontal axis). We then fit our input-output model for different values of the barrier gate voltages and extract the tunnel coupling for each line cut. The extracted tunnel couplings can be well fit by a linear dependence (see Fig. 6) allowing us to continuously convert the vertical axis from units of barrier gate voltage to tunnel coupling.

[1] J. Yoneda, K. Takeda, T. Otsuka, T. Nakajima, M. R. Delbecq, G. Allison, T. Honda, T. Kodera, S. Oda, Y. Hoshi, N. Usami, K. M. Itoh, and S. Tarucha, A quantum-dot spin qubit with coherence limited by charge noise and fidelity higher than 99.9\%, Nat. Nanotechnol. 13, 102 (2018).

[2] C. H. Yang, K. W. Chan, R. Harper, W. Huang, T. Evans, J. C. C. Hwang, B. Hensen, A. Laucht, T. Tanttu, F. E. Hudson, S. T. Flammia, K. M. Itoh, A. Morello, S. D. Bartlett, and A. S. Dzurak, Silicon qubit fidelities approaching incoherent noise limits via pulse engineering, Nat. Electron. 2, 151 (2019).

[3] R. W. Andrews, C. Jones, M. D. Reed, A. M. Jones, S. D. Ha, M. P. Jura, J. Kerckhoff, M. Levendorf, S. Meenehan, S. T. Merkel, A. Smith, B. Sun, A. J. Weinstein, M. T. Rakher, T. D. Ladd, and M. G. Borselli, Quantifying error and leakage in an encoded $\mathrm{Si} / \mathrm{SiGe}$ triple-dot qubit, Nat. Nanotechnol. 14, 747 (2019).

[4] W. Huang, C. H. Yang, K. W. Chan, T. Tanttu, B. Hensen, R. C. C. Leon, M. A. Fogarty, J. C. C. Hwang, F. E. Hudson, K. M. Itoh, A. Morello, A. Laucht, and A. S. Dzurak, Fidelity benchmarks for two-qubit gates in silicon, Nature (London) 569, 532 (2019).

[5] X. Xue, T. Watson, J. Helsen, D. Ward, D. Savage, M. Lagally, S. Coppersmith, M. Eriksson, S. Wehner, and L. Vandersypen, Benchmarking Gate Fidelities in a Si/SiGe Two-Qubit Device, Phys. Rev. X 9, 021011 (2019).

[6] F. Schäffler, High-mobility Si and Ge structures, Semicond. Sci. Technol. 12, 1515 (1997).

[7] P. W. Deelman, L. F. Edge, and C. A. Jackson, Metamorphic materials for quantum computing, MRS Bull. 41, 224 (2016).

[8] S. J. Angus, A. J. Ferguson, A. S. Dzurak, and R. G. Clark, Gate-defined quantum dots in intrinsic silicon, Nano Lett. 7, 2051 (2007).

[9] M. G. Borselli, K. Eng, R. S. Ross, T. M. Hazard, K. S. Holabird, B. Huang, A. A. Kiselev, P. W. Deelman, L. D. Warren, I. Milosavljevic, A. E. Schmitz, M. Sokolich, M. F. Gyure, and A. T. Hunter, Undoped accumulation-mode $\mathrm{Si} / \mathrm{SiGe}$ quantum dots, Nanotechnology 26, 375202 (2015).

[10] D. M. Zajac, T. M. Hazard, X. Mi, E. Nielsen, and J. R. Petta, Scalable Gate Architecture for a One-Dimensional Array of Semiconductor Spin Qubits, Phys. Rev. Appl. 6, 054013 (2016)

[11] W. I. L. Lawrie, H. G. J. Eenink, N. W. Hendrickx, J. M. Boter, L. Petit, S. V. Amitonov, M. Lodari, B. Paquelet Wuetz, C. Volk, S. G. J. Philips, G. Droulers, N. Kalhor, F. van Riggelen, D. Brousse, A. Sammak, L. M. K. Vandersypen, G. Scappucci, and M. Veldhorst, Quantum dot arrays in silicon and germanium, Appl. Phys. Lett. 116, 080501 (2020).

[12] A. R. Mills, D. M. Zajac, M. J. Gullans, F. J. Schupp, T. M. Hazard, and J. R. Petta, Shuttling a single charge across a one-dimensional array of silicon quantum dots, Nat. Commun. 10, 1063 (2019).

[13] F. A. Zwanenburg, A. S. Dzurak, A. Morello, M. Y. Simmons, L. C. L. Hollenberg, G. Klimeck, S. Rogge, S. N. Coppersmith, and M. A. Eriksson, Silicon quantum electronics, Rev. Mod. Phys. 85, 961 (2013).

[14] S. Goswami, K. A. Slinker, M. Friesen, L. M. McGuire, J. L. Truitt, C. Tahan, L. J. Klein, J. O. Chu, P. M. Mooney, D. W. van der Weide, R. Joynt, S. N. Coppersmith, and M. A. Eriksson, Controllable valley splitting in silicon quantum devices, Nat. Phys. 3, 41 (2007).

[15] N. E. Penthorn, J. S. Schoenfield, J. D. Rooney, L. F. Edge, and H. Jiang, Two-axis quantum control of a fast valley qubit in silicon, npj Quantum Inf. 5, 1 (2019).

[16] M. Friesen, M. A. Eriksson, and S. N. Coppersmith, Magnetic field dependence of valley splitting in realistic $\mathrm{Si} / \mathrm{SiGe}$ quantum wells, Appl. Phys. Lett. 89, 202106 (2006).

[17] M. Friesen and S. N. Coppersmith, Theory of valley-orbit coupling in a $\mathrm{Si} / \mathrm{SiGe}$ quantum dot, Phys. Rev. B 81, 115324 (2010).

[18] B. Tariq and X. Hu, Effects of interface steps on the valleyorbit coupling in a $\mathrm{Si} / \mathrm{SiGe}$ quantum dot, Phys. Rev. B 100, 125309 (2019). 
[19] J. M. Elzerman, R. Hanson, L. H. W. Van Beveren, B. Witkamp, L. M. K. Vandersypen, and L. P. Kouwenhoven, Single-shot read-out of an individual electron spin in a quantum dot, Nature (London) 430, 431 (2004).

[20] C. H. Yang, W. H. Lim, N. S. Lai, A. Rossi, A. Morello, and A. S. Dzurak, Orbital and valley state spectra of a few-electron silicon quantum dot, Phys. Rev. B 86, 115319 (2012).

[21] L. Petit, J. Boter, H. Eenink, G. Droulers, M. Tagliaferri, R. Li, D. Franke, K. Singh, J. Clarke, R. Schouten, V. Dobrovitski, L. Vandersypen, and M. Veldhorst, Spin Lifetime and Charge Noise in Hot Silicon Quantum Dot Qubits, Phys. Rev. Lett. 121, 076801 (2018).

[22] F. Borjans, D. M. Zajac, T. M. Hazard, and J. R. Petta, Single-Spin Relaxation in a Synthetic Spin-Orbit Field, Phys. Rev. Appl. 11, 044063 (2019).

[23] X. Zhang, R.-Z. Hu, H.-O. Li, F.-M. Jing, Y. Zhou, R.-L. Ma, M. Ni, G. Luo, G. Cao, G.-L. Wang, X. Hu, H.-W. Jiang, G.-C. Guo, and G.-P. Guo, Giant Anisotropy of Spin Relaxation and Spin-Valley Mixing in a Silicon Quantum Dot, Phys. Rev. Lett. 124, 257701 (2020).

[24] M. Friesen, S. Chutia, C. Tahan, and S. N. Coppersmith, Valley splitting theory of $\mathrm{SiGe} / \mathrm{Si} / \mathrm{SiGe}$ quantum wells, Phys. Rev. B 75, 115318 (2007).

[25] D. Culcer, X. Hu, and S. Das Sarma, Interface roughness, valley-orbit coupling, and valley manipulation in quantum dots, Phys. Rev. B 82, 205315 (2010).

[26] Z. Jiang, N. Kharche, T. Boykin, and G. Klimeck, Effects of interface disorder on valley splitting in $\mathrm{SiGe} / \mathrm{Si} / \mathrm{SiGe}$ quantum wells, Appl. Phys. Lett. 100, 103502 (2012).

[27] S. F. Neyens, R. H. Foote, B. Thorgrimsson, T. J. Knapp, T. McJunkin, L. M. K. Vandersypen, P. Amin, N. K. Thomas, J. S. Clarke, D. E. Savage, M. G. Lagally, M. Friesen, S. N. Coppersmith, and M. A. Eriksson, The critical role of substrate disorder in valley splitting in $\mathrm{Si}$ quantum wells, Appl. Phys. Lett. 112, 243107 (2018).

[28] M. G. Borselli, R. S. Ross, A. A. Kiselev, E. T. Croke, K. S. Holabird, P. W. Deelman, L. D. Warren, I. AlvaradoRodriguez, I. Milosavljevic, F. C. Ku, W. S. Wong, A. E. Schmitz, M. Sokolich, M. F. Gyure, and A. T. Hunter, Measurement of valley splitting in high-symmetry $\mathrm{Si} / \mathrm{SiGe}$ quantum dots, Appl. Phys. Lett. 98, 123118 (2011).

[29] D. M. Zajac, T. M. Hazard, X. Mi, K. Wang, and J. R. Petta, A reconfigurable gate architecture for $\mathrm{Si} / \mathrm{SiGe}$ quantum dots, Appl. Phys. Lett. 106, 223507 (2015).

[30] X. Mi, C. G. Péterfalvi, G. Burkard, and J. R. Petta, HighResolution Valley Spectroscopy of Si Quantum Dots, Phys. Rev. Lett. 119, 176803 (2017).

[31] R. Ferdous, E. Kawakami, P. Scarlino, M. P. Nowak, D. R. Ward, D. E. Savage, M. G. Lagally, S. N. Coppersmith, M. Friesen, M. A. Eriksson, L. M. K. Vandersypen, and R. Rahman, Valley dependent anisotropic spin splitting in silicon quantum dots, npj Quantum Inf. 4, 1 (2018).

[32] A. Hollmann, T. Struck, V. Langrock, A. Schmidbauer, F. Schauer, T. Leonhardt, K. Sawano, H. Riemann, N. V. Abrosimov, D. Bougeard, and L. R. Schreiber, Large, Tunable Valley Splitting and Single-Spin Relaxation Mechanisms in a $\mathrm{Si} / \mathrm{Si}_{x} \mathrm{Ge}_{1-x}$ Quantum Dot, Phys. Rev. Appl. 13, 034068 (2020).
[33] E. H. Chen, K. Raach, A. Pan, A. A. Kiselev, E. Acuna, J. Z. Blumoff, T. Brecht, M. D. Choi, W. Ha, D. R. Hulbert, M. P. Jura, T. E. Keating, R. Noah, B. Sun, B. J. Thomas, M. G. Borselli, C. A. C. Jackson, M. T. Rakher, and R. S. Ross, Detuning Axis Pulsed Spectroscopy of Valley-Orbital States in Si/Si-Ge Quantum Dots, Phys. Rev. Appl. 15, 044033 (2021).

[34] C. H. Yang, A. Rossi, R. Ruskov, N. S. Lai, F. A. Mohiyaddin, S. Lee, C. Tahan, G. Klimeck, A. Morello, and A. S. Dzurak, Spin-valley lifetimes in a silicon quantum dot with tunable valley splitting, Nat. Commun. 4, 2069 (2013).

[35] J. K. Gamble, P. Harvey-Collard, N. T. Jacobson, A. D. Baczewski, E. Nielsen, L. Maurer, I. Montaño, M. Rudolph, M. S. Carroll, C. H. Yang, A. Rossi, A. S. Dzurak, and R. P. Muller, Valley splitting of single-electron Si MOS quantum dots, Appl. Phys. Lett. 109, 253101 (2016).

[36] C. H. Yang, R. C. C. Leon, J. C. C. Hwang, A. Saraiva, T. Tanttu, W. Huang, J. Camirand Lemyre, K. W. Chan, K. Y. Tan, F. E. Hudson, K. M. Itoh, A. Morello, M. Pioro-Ladrière, A. Laucht, and A. S. Dzurak, Operation of a silicon quantum processor unit cell above one kelvin, Nature (London) 580, 350 (2020).

[37] S.-y. Shiau, S. Chutia, and R. Joynt, Valley Kondo effect in silicon quantum dots, Phys. Rev. B 75, 195345 (2007).

[38] D. Culcer, L. Cywiński, Q. Li, X. Hu, and S. Das Sarma, Quantum dot spin qubits in silicon: Multivalley physics, Phys. Rev. B 82, 155312 (2010).

[39] J. K. Gamble, M. A. Eriksson, S. N. Coppersmith, and M. Friesen, Disorder-induced valley-orbit hybrid states in Si quantum dots, Phys. Rev. B 88, 035310 (2013).

[40] M. Russ, C. G. Péterfalvi, and G. Burkard, Theory of valley-resolved spectroscopy of a Si triple quantum dot coupled to a microwave resonator, J. Phys.: Condens. Matter 32, 165301 (2020).

[41] L. Landau, Theory of energy transfer. II, Phys. Z. Sowjetunion 2, 46 (1932).

[42] X. Zhao and X. Hu, Coherent electron transport in silicon quantum dots, arXiv:1803.00749 (2018).

[43] F. Ginzel, A. R. Mills, J. R. Petta, and G. Burkard, Spin shuttling in a silicon double quantum dot, Phys. Rev. B 102, 195418 (2020).

[44] B. Koiller, X. Hu, and S. Das Sarma, Exchange in SiliconBased Quantum Computer Architecture, Phys. Rev. Lett. 88, 027903 (2001).

[45] A. Wallraff, D. I. Schuster, A. Blais, L. Frunzio, R.S. Huang, J. Majer, S. Kumar, S. M. Girvin, and R. J. Schoelkopf, Strong coupling of a single photon to a superconducting qubit using circuit quantum electrodynamics, Nature 431, 162 (2004).

[46] K. D. Petersson, L. W. McFaul, M. D. Schroer, M. Jung, J. M. Taylor, A. A. Houck, and J. R. Petta, Circuit quantum electrodynamics with a spin qubit, Nature (London) 490 , 380 (2012).

[47] T. Frey, P. J. Leek, M. Beck, A. Blais, T. Ihn, K. Ensslin, and A. Wallraff, Dipole Coupling of a Double Quantum Dot to a Microwave Resonator, Phys. Rev. Lett. 108, 046807 (2012).

[48] J. J. Viennot, M. C. Dartiailh, A. Cottet, and T. Kontos, Coherent coupling of a single spin to microwave cavity photons, Science 349, 408 (2015). 
[49] A. Stockklauser, P. Scarlino, J. V. Koski, S. Gasparinetti, C. K. Andersen, C. Reichl, W. Wegscheider, T. Ihn, K. Ensslin, and A. Wallraff, Strong Coupling Cavity QED with Gate-Defined Double Quantum Dots Enabled by a High Impedance Resonator, Phys. Rev. X 7, 011030 (2017).

[50] X. Mi, J. V. Cady, D. M. Zajac, P. W. Deelman, and J. R. Petta, Strong coupling of a single electron in silicon to a microwave photon, Science 355, 156 (2017).

[51] X. Mi, M. Benito, S. Putz, D. M. Zajac, J. M. Taylor, G. Burkard, and J. R. Petta, A coherent spin-photon interface in silicon, Nature (London) 555, 599 (2018).

[52] N. Samkharadze, G. Zheng, N. Kalhor, D. Brousse, A. Sammak, U. C. Mendes, A. Blais, G. Scappucci, and L. M. K. Vandersypen, Strong spin-photon coupling in silicon, Science 359, 1123 (2018).

[53] F. Borjans, X. G. Croot, X. Mi, M. J. Gullans, and J. R. Petta, Resonant microwave-mediated interactions between distant electron spins, Nature (London) 577, 195 (2020).

[54] A. J. Landig, J. V. Koski, P. Scarlino, U. C. Mendes, A. Blais, C. Reichl, W. Wegscheider, A. Wallraff, K. Ensslin, and T. Ihn, Coherent spin-photon coupling using a resonant exchange qubit, Nature (London) 560, 179 (2018).

[55] T. Ando, A. B. Fowler, and F. Stern, Electronic properties of two-dimensional systems, Rev. Mod. Phys. 54, 437 (1982).
[56] G. Burkard and J. R. Petta, Dispersive readout of valley splittings in cavity-coupled silicon quantum dots, Phys. Rev. B 94, 195305 (2016).

[57] F. Borjans, X. Croot, S. Putz, X. Mi, S. M. Quinn, A. Pan, J. Kerckhoff, E. J. Pritchett, C. A. Jackson, L. F. Edge, R. S. Ross, T. D. Ladd, M. G. Borselli, M. F. Gyure, and J. R. Petta, Split-gate cavity coupler for silicon circuit quantum electrodynamics, Appl. Phys. Lett. 116, 234001 (2020).

[58] X. Mi, J. V. Cady, D. M. Zajac, J. Stehlik, L. F. Edge, and J. R. Petta, Circuit quantum electrodynamics architecture for gate-defined quantum dots in silicon, Appl. Phys. Lett. 110, 043502 (2017).

[59] M. Benito, X. Mi, J. M. Taylor, J. R. Petta, and G. Burkard, Input-output theory for spin-photon coupling in Si double quantum dots, Phys. Rev. B 96, 235434 (2017).

[60] M. F. Gonzalez-Zalba, S. Barraud, A. J. Ferguson, and A. C. Betz, Probing the limits of gate-based charge sensing, Nat. Commun. 6, 6084 (2015).

[61] B. M. Maune, M. G. Borselli, B. Huang, T. D. Ladd, P. W. Deelman, K. S. Holabird, A. A. Kiselev, I. AlvaradoRodriguez, R. S. Ross, A. E. Schmitz, M. Sokolich, C. A. Watson, M. F. Gyure, and A. T. Hunter, Coherent singlettriplet oscillations in a silicon-based double quantum dot, Nature (London) 481, 344 (2012). 